\title{
Introducing emotions in the architectural design process
}

\author{
F. Juan-Vidal*, S. Iñarra-Abad* \\ * Departamento de Expresión Gráfica Arquitectónica. Universidad Politécnica de Valencia (UPV)
}

\begin{abstract}
D images or architectural renders are expressive tools widely used by architectural students to communicate their projects. Not only do these images have the task of transmitting the formal and functional characteristics of the project, but also need to evoke in users the sort of feelings and emotions which can only be aroused by experiencing the architecture. An experiment we undertook enabled us to find that these emotions can be quantified and may relate to the design parameters of the image, moreover there are also substantial differences in the valuation between architects and non-architects. Thus, the aim of this paper is to incorporate the results of our research on the learning of this graphical tool into the learning of architecture. Therefore we have established a teaching methodology in which the images are not only employed to show the final result of design, but are also incorporated into the creative process to investigate emotional and aesthetic responses of the user of the designed space..
\end{abstract}

Keywords: architectural design process, architectural rendering, emotions, subjective evaluation

\section{Introduction}

Learning the process of architectural conception has always been a complex task. Nowadays, its intrinsic difficulty is added to by an epistemological slippage of the concept of "architectural space" as well as by the impact of new computer-based tools in the virtual representation of architecture.

\section{The process of designing}

It is said that drawing "represents" architecture in the sense that it specifies its anticipation, its imitation, or even, in some senses, its substitution. Authors such as E. H. Gombrich ('Art and Illusion', 1959) show that the level of representation of a drawing depends less on the degree of imagination or imitation, rather the use made of it as a "substitution" of the physical reality. Whereas the graphical object it evokes is made possible by its 'illusion'.

Once the idea of the project has been reached, the architect needs to involve others in it. The architect is usually required to communicate their project to two different parties; on the one hand to the developer or the party which requires the project and on the other to the builder or agent who will construct it.

The promoter is the driver and initiator of the project. For them, the intention will be of an illustrative nature, with the purpose of "presenting" the project, expressing it so it quickly communicates the idea. It generally consists of pure anticipatory and figurative representation of the architectural object. Following the tradition that began in the Italian Renaissance, for this purpose the architect resorts to the use of realistic visions based on perspective and in more or less gimmicky graphical techniques (textures, lighting and shadows) or mock-ups or three-dimensional scale models. Nowadays, architectural render is used for this function.

In each case, the concept of these presentations depends not so much on the choice of tool, rather on the success in transmitting the 'essence' of the architecture. Perhaps it is less about showing the form rather it is about being able to express everything else through it. 


\section{The 'essence' of architecture}

During the latter third of the 20th Century the Modern theories of supremacy of physical space at the core of architecture started to be challenged, above all as a reflection of new waves of thought beginning a conceptual change in the understanding of art. With the existentialism of Heidegger's (1889 - 1976) 'Being-inthe-world', the world is no longer a collection of objects in which the individual finds himself subject, rather as the fundamental characteristic of human existence. In first place will be 'my world' rather than the presumed objective world as proposed by science.

Authors such as C. Norebrg-Schulz ('Intentions in Architecture', 1963) in keeping with these lines of thought propose the substitution of the idea of 'interior space' with the concept of 'existential space'. Space is defined as an existential dimension of an individual whose essence is given from 'place' and not from the physical dimensions

Architecture accommodates human activities. It encompasses their home life, work, study, worship, recreation... Not only this, but it also provides order and comfort. However when architecture, as well as satisfying these specific human specifications, responds to the existential experience of 'dwelling', it creates 'places' and is also a source of well-being.

Now, spaces are not the most important things; rather the environments. Small details perceived by the senses become worthy (the early morning light through a Mallorcan shutter, the rustling of a poplar grove, the smell of dew on a lawn, the creaking of footsteps on antique oak-wood floorboards). Emotions now come into play. The differences between doing architecture and the form of living it become blurred. Design now is not only the search of a particular aesthetic but the provision of places and environments which accommodate the emotions of others.

\section{Expressing and conceiving places}

However, if limitations are found in Modern theories of representing the spatial essence of architecture: What problems do today's architects face in representing environments, fantastic places or habitats in their projects? How to represent these feelings and emotions? In what way can the existential component of the architecture be expressed and presented? Without doubt, there is no possibility of drawing 'wellbeing'.

Of course, if we pay attention to those who hold that the architectural element, with all its material complexity, conventional, spatial and existentialistic, can only be represented by itself. To try substitution (representation) would be little more than a pipe-dream and we would have to conclude that it is not possible to represent the existential component of architecture. However, in contrary to this, if we accept the structuralist's view that it is possible to get close to a comprehension of an architectural element through its components or parts; If give up in our attempt to take in the whole complexity in one go and we apply the method of breaking up; If we accept the whole and admit that a representation can only try to create a substitute capable of a specific, partial result, then we have to admit that it is possible to provoke in the imagination the illusion of a material, form, space and even attain similar sensations to those which are felt in a place or during an experience, even though it is in a limited and fragmented form. 
In this way the illustration may function as a substitute of the existential space that contributes to architectural projects and evoke some of the emotions which arise from occupying them. For this motive, besides being a medium for the illustrative presentation of the final result of the project it may also be an active and effective means, in the initial phases, of orientating the process of conception of spaces which favour certain emotions.

\section{Material and Methods}

Nowadays the architectural render is an indispensable medium of expression for the communication of an architectural project. The rapid advance of computer programs for digital modelling has allowed architects to produce sophisticated images as a valuable tool to entice viewers. The emotional impressions evoked in the viewer by these images produce a more or less favourable opinion about the space that the image represents.

The emotional process by which we analyse our environment is a complex one, in which our perception and cognition take part. The personality and psychological state of the observer, their reactions to their circumstances, previous experiences of spaces, etc. also have a role in the process. Given the complexity of the whole process, Nasar (1994) proposed a probabilistic model of the aesthetic response which is summarised in figure 1.

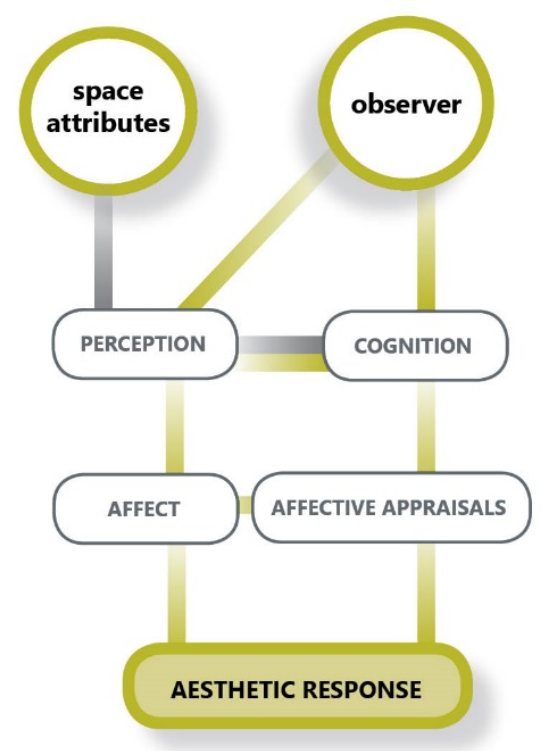

Figure 1. Probabilistic model of aesthetic response to the built environment.

According to the scheme, when we try to improve the value that observers will put on a space represented by a digital image, or try to obtain a better aesthetic response from an image, it is important to recognise and identify which emotions the observer felt before that particular observation.

In the Departamento de Expresión Gráfica de la Escuela de Arquitectura de Valencia, we have conducted an experiment to determine the emotional state of an observer evoked by an image during the process of assessment of an architectural project and, in turn, how this emotional state relates to the overall appraisal of the project. 
The method was based on Osgood's semantic differential (Osgood et al, 1957). A field study was carried out on a sample of 217 subjects (104 architects and 113 nonarchitects) who were asked to express their opinions on 48 renders of architectural spaces.

The results show that the perception of the architectural render can be expressed through 6 independent concepts or semantic axes, which explains 55\% of the variance in the sampled perception. These axes or factors are, by order of explained variance: tranquillity - well-being; innovation - futuristic; happiness - warmth; nostalgia romanticism; functionality and monumentality - luxury.

The results also show that, for both groups, the success of a render is associated with the emotions of innovation - futuristic and tranquillity - well-being.

Figure 2 shows the relationship between these semantic axes with global assessment. Also the results show that there are significant differences between the architects and non-architects. Thus what is innovative or evokes well-being in one group is not so in the other group. In general, architects are more critical in their assessments (Llinares and Iñarra, 2014).
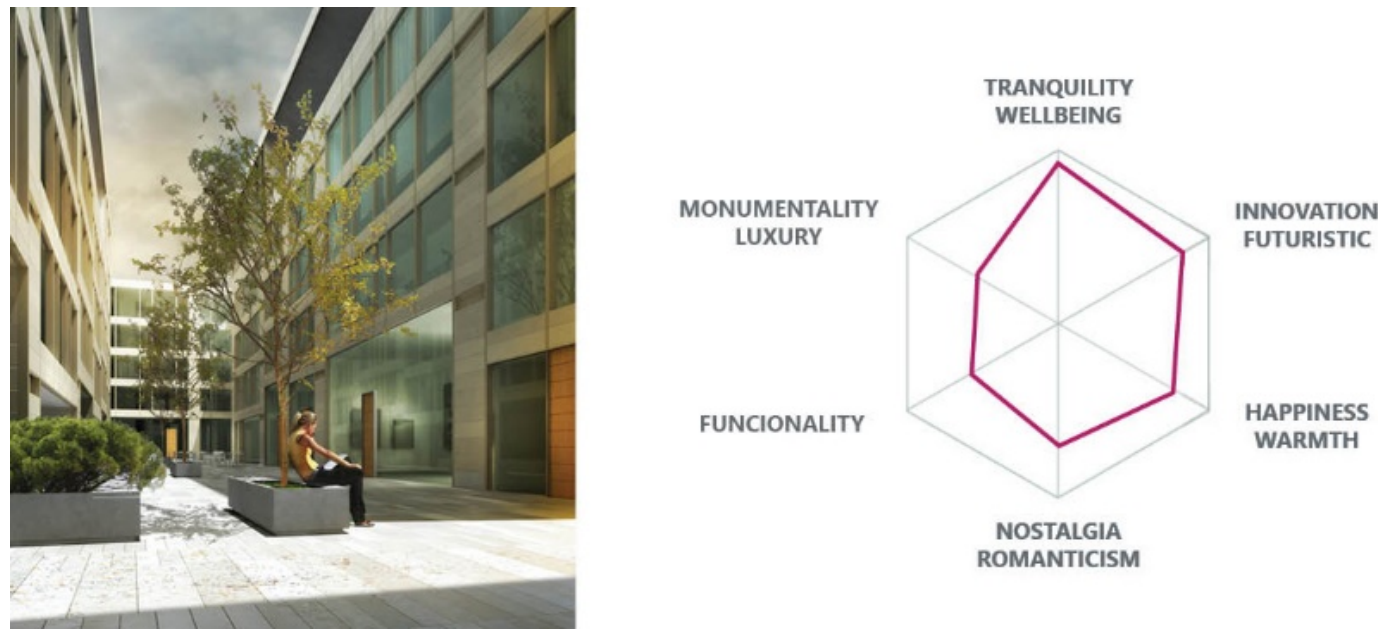

Figure 2. Relation of semantic axes with global assessment

These results provide evidence that it is necessary for the student of architecture, in the process of the conception of the project besides the formal conditions and aesthetics, to also take into account the emotions that their project will promote in the future user of the space. In order to incorporate this new determinant in the design process, besides the architect's own intuition and feelings, there are methods which have been developed in the field of product design which can quantify the emotions experienced by the user and translate them into particular criteria of a space.

We have designed a working method with the objective of using the results of this educational study in the field of architectural graphic expression to apply them to different subjects of architectural study.

\section{Materials}

In order that the architectural student can investigate the emotions that the representations of their projects will awaken in the observer, questionnaires developed from the results of our experimental work will be employed. In this way, we consider that the six semantic concepts obtained (tranquillity - wellbeing; innovation futuristic; happiness - warmth; nostalgia - romanticism, functionality and 
monumentality - luxury) will serve as a basis for the development of the questionnaires.

The student will prepare 3 render images (or virtual perspectives) of the architectural project for each of the distinct phases of the process: 1 . of conception; 2 . of reflection; and 3. of presentation (a total of 9 rendered images per student). These images will be subject to observation by a sample of subjects, ensuring an acceptable level of statistical power and statistical significance for this type of comparison. The criteria will involve obtaining at least 6 records for each variable and project.

In the fieldwork, subjective information for each image will be collected as a function of the semantic axes evaluated on a Likert scale of 5 levels (totally agree, partially agree, indifferent, partially disagree and totally disagree). Also the participant will be asked to express their global assessment.

Given the difference in the answers between the architects and non-architects, it is suggested that the questionnaire be conducted with two different groups of participants: on one hand architecture students and on the other a determined number of external individuals from various backgrounds outside the field of architecture.

\section{Methods}

The study will be divided into three parts corresponding with the three phases in the process of the project (conception, reflection and presentation). A specific questionnaire will be carried out at the end of each phase.

In the first phase the students will be organised in sub-groups of 4 , in the second phase in sub-groups of 2 and in the third they will work individually. Each sub-group will work on a project with the same given theme. Supposing there are a group of 20 students, there will be 5 projects in the first phase, 10 in the second phase and 20 in the third.

At the end of the first phase we will have 5 questionnaires (one for each project), each one comprising of 3 render images. In the same way, by the end of the second phase, there will be 10 questionnaires. Finally, at the end of the study, there will be 20 questionnaires.

With this method it is hoped to generate a feedback process using the results of the questionnaires in two directions: one horizontal, in each phase where conclusions will be drawn for compassion between projects; and the other vertical, in each project, allowing the reshaping the results and increasing as much the level of development as the degree of personalisation of each project.

\section{Results and Discussion}

In order to extract and analyse the data obtained from the questionnaires, the students will be given a basic knowledge of statistics.

The results obtained in each phase of the creation of the project will allow a classification of the images into two levels: on one hand based on the emotions evoked in the observer and on the other hand, based on the assessment of the project that the image represent.

The comparison between the different modes of three dimensional representation for the same project will allow the students to establish conclusions as to how the design 
parameters evoke different emotions. For example, the absence of people increases the sensation of nostalgia, the presence of nature evokes greater tranquillity, blue sky awakens happiness...

Knowing at the same time which emotions are related to a better valuation of the project, the student will be able to work this into the project to give rise to better success in the later phases of the project.

Analysis of the differences in opinion between the students and people outside of the architecture field will allow the student to understand the semantic distance which exists between the two groups, which has previously been tested in different experimental works (Devlin and Nasar, 1989; Gifford, 2002).

\section{Conclusions}

The architect creates living spaces and these must awake in the user favourable emotions that promote the activity for which the space has been created: well-being in the home; security in urban spaces; sobriety in work places; monumentalism in the symbols of the city, etc.

Until now, the capacity of the architect to delve into these aspects has been developed through their own experience. Our proposal is an attempt to equip future architects with experimentally validated tools to investigate the emotional response of the user and to incorporate this response into the design of the architectural space.

This contribution to the education of future architects will allow the definition of new design criteria for spaces oriented around the user at the emotional level, achieving a more human and efficient architecture, resulting in a better quality of life for the inhabitants.

\section{References}

Devlin, K. \& Nasar, J. (1989) The beauty and the beast: some preliminary comparisons of "high" versus "popular" residential architecture and public versus architect judgments of same. Journal of Environment Psychology, 9, 333-344.

Gifford, R. (2002) Why architects and laypersons judge buildings differently: cognitive properties and physical bases. Journal Architecture Planning Research, 19 (2), 131-148.

Gombrich, E. (1959). Art and ilusion. Consulted the Spanish edition of Gustavo Gili, Barcelona, 1979.

Juan-Vidal F. (2006). Figurando Lugares. Funciones del dibujo en la producción actual de Arquitectura. Universidad de Sevilla.

Llinares, C., \& Iñarra, S. (2014). Human factors in computer simulations of urban environment. Differences between architects and non-architects' assessments. Displays, 35(3), 126-140.

Nasar, J. (1994) Urban design aesthetics: The evaluative qualities of building exteriors. Environment \& Behaviour, 26 (3), 377-401.

Norberg-Schulz, C. (1963). Intentions in architecture. Consulted the Spanish version by J. Sainz y F. González Fernández. Ed. Gustavo Gili. Barcelona, 1979.

Osgood C.E., Suci G.J. \& Tannenbaum P.H. (1957) The Measurement of Meaning. Illinois: University of Illinois Press. USA. 